\title{
EDQM-HC State of Matter Terminology
}

National Cancer Institute

\section{Source}

National Cancer Institute. EDQM-HC State of Matter Terminology. NCI Thesaurus. Code C150116.

Terminology subset about physical conditions describing the molecular form of a product. Note: State of matter is used to group basic dose forms according to their physical properties. 